\title{
Aus der Arbeit des FaDaF
}

Bericht über die 30. Jahrestagung Deutsch als Fremdsprache an der Ludwig-Maximilians-Universität München vom 30. Mai bis 1. Juni 2002

(Der Sammelband zur Tagung wird als Heft 70 mit dem Titel Mehrsprachigkeit in der Reihe "Materialien Deutsch als Fremdsprache (MatDaF)« im Frühjahr 2003 erscheinen.)

Die 30. Jahrestagung Deutsch als Fremdsprache des Fachverbandes Deutsch als Fremdsprache $(\mathrm{FaDaF})$ fand vom 30. Mai bis 1. Juni 2002 an der LudwigMaximilians-Universität München statt und wurde vom Vorstand des Fachverbandes gemeinsam mit dem Institut für Deutsch als Fremdsprache/Transnationale Germanistik der Ludwig-Maximilians-Universität München in 4 Themenschwerpunkten und dem Forum Deutsch als Fremdsprache vorbereitet und durchgeführt. Selbstverständlich gehörten auch zu dieser Tagung wieder eine umfangreiche Präsentation von Fachbüchern durch Verlage, die Mitgliederversammlung des FaDaF, Treffen von Lehrkräften für $\mathrm{DaF}$ und gesellige Rahmenveranstaltungen.

(Interessierte können Informationen über die Ergebnisse einzelner Treffen erhalten bei der Geschäftsstelle des Fachverbandes Deutsch als Fremdsprache beim Fachsprachenzentrum der Universität Hannover, Am Welfengarten 1, 30167 Hannover, Tel. 0511-762-5716; Fax: 0511762-4008; e-mail: fadaf-lau@fsz.uni-hannover.de.)
Themenschwerpunkt 1: Die Vielperspektivität in Literatur und Literaturvermittlung

(Uwe Koreik, Hannover; Michael Ewert, München; Willie van Peer, München; Ursula Renate Riedner, München)

Bei dieser Tagung stand zum ersten Mal seit Jahren wieder das Thema Literatur im DaF-Unterricht im Vordergrund. »Die Vielperspektivität in Literatur und Literaturvermittlung « war der genaue Titel des Themenschwerpunktes 1 , der bei den Tagungsteilnehmern auf lebhaftes Interesse stieß. Zwischen fünfzig und achtzig Tagungsbesucher fanden sich regelmäßig zu den auf 30 Minuten begrenzten Vorträgen ein, und - das war das Besondere - wirkten in großer Zahl engagiert bei den jeweils folgenden Diskussionen eine weitere halbe Stunde mit.

Den Anfang machte der Münchener Lehrstuhlinhaber Willie van Peer, der gemeinsam mit Maria-Savato Theodoridou durchgeführte Untersuchungen zur "Pragmatik des Lesens in unterschiedlichen Kulturen " vorstellte. Anhand des Vergleichs von Reaktionen deutscher wie nicht-deutscher Rezipienten auf kurze literarische Werke wurde aufgezeigt, daß entgegen der vielfach angenommenen stark kulturell bestimmten Sichtweise die Verarbeitung literarischer Texte zum Teil von Regeln gesteuert werde, »die vermutlich auf einer quasi-universellen Grundlage beruhen«.

Stefan Hajduk (Poona) entwickelte auf der Basis seiner Erfahrungen mit der kulturfremden Lektüre von Fontanes Effi Briest in Indien weitreichende und weiterführende Überlegungen $\mathrm{zu}$ einer literari- 
schen Hermeneutik in Auseinandersetzung mit bisherigen theoretischen Ansätzen. Bereits in der anschließenden Diskussion wurde klar, daß zahlreiche der vorgetragenen Argumentationszusammenhänge erst nach einer eingehenden Lektüre seines Textes entsprechend gewürdigt werden können.

Manfred Schewe (Cork) stellte die kritische Frage nach der gängigen Praxis der Literaturvermittlung an ausländischen Hochschulen und betonte auch anhand des Beispiels von Thomas Hürlimanns Das Lied der Heimat die Notwendigkeit eines verstärkten Ernstnehmens »einer künstlerischen Orientierung im Bereich Literaturvermittlung «, indem er die bisherige Sozialisation zur Literatur einer Sozialisation durch Literatur gegenüberstellte.

Harald Tanzer (Regensburg) beleuchtete den Funktionswandel von Fremdheit in der deutschsprachigen Prosa türkischer Autoren der neunziger Jahre. In vielen Texten sei nicht mehr wie in den siebziger und achtziger Jahren eine literarische Auseinandersetzung mit der Fremdheit der Bundesrepublik zu beobachten, sondern bilde die Fremderfahrung nun den Rahmen für Selbsterfahrung, wobei Deutschland Mittel und Gegenstand einer deutsch-türkischen Identitätsdiskussion darstelle.

Klaus Schenk (Prag) rückte Überlegungen zur Literatur als Ausdruck von Fremdheit und displacement in den Vordergrund. U. a. an Beispielen aus dem Werk des syrischen Autors Rafik Schami betonte er, daß es im Bereich des Fremdsprachenerwerbs gelte, den bisherigen Lesekanon auszuweiten. Die Fremdsprache aus der Perspektive des Fremden zu beleuchten, biete die Möglichkeit einer Didaktik als displacement, die weniger versuche, deutsche Sprache und Kultur zu vermitteln als sich die Differenzen von
Kulturen und Sprachen zum Medium zu machen.

Swantje Ehlers (Gießen) lenkte den Fokus auf die Beziehungen zwischen Selbstund Fremdbild am Beispiel der Bilder von Afrika in der Kinder- und Jugendliteratur. Seit den neunziger Jahren diene die fremde Welt nicht mehr unbedingt als exotische Kulisse für den Auftritt des edlen Europäers, sondern veränderten sich die Perspektiven, indem »der Blickpunkt ins Innere der fremden Kultur verschoben« werde. Dadurch seien die Bilder in dieser Literatur, indem sie aus Binnenperspektiven resultieren, potentiell Fenster zu anderen Kulturen (Clifford 1988), welche somit »Einblick gewähren und fremde Lebensweisen und Weltbilder in ihrer Eigenheit verständlich machen «.

Uta Aifan (Stuttgart) beschäftigte sich mit literarischen Eigenbildern im Spiegel der Fremdwahrnehmung deutsch-arabischer Autoren, insbesondere mit »Grenzgängerautoren, die im spannungsgeladenen Beziehungsdreieck deutsch-jüdisch-arabischer Beziehungen anzusiedeln sind «. Werke von Adel Karasholi und Rafik Shami seien dadurch gekennzeichnet, daß der Blick auf das Eigene von ihrer Wahrnehmung des Fremden beeinflußt werde, wodurch insbesondere in der interkulturellen Vermittlung ein Umgang mit der Pluralität der Bilder und Blickwinkel geübt und eine Reduktion der Analyse auf Landschaftsmerkmale oder positive und negative Darstellungen vermieden werden könne.

Ursula Renate Riedner (München), Mitkoordinatorin des Themenschwerpunkts, lenkte den Blick auf Karl Valentins und Liesl Karlstadts »Theater in der Vorstadt", wobei deutlich wurde, daß Stücke Valentins und der Schauspielerin und häufigen Mitautorin Karlstadt nicht nur von Intellektuellen zu Beginn des 20. Jahrhunderts geschätzt wurden, sondern sich seit An- 
fang der 60er Jahre großer Beliebtheit erfreuen. Sie wurden nicht nur in West- und Ostdeutschland, sondern auch in zahlreichen Ländern Europas, in den USA und in Brasilien erfolgreich aufgeführt. Die Wirksamkeit von Komik sei zwar von den auch kulturell geprägten - Erwartungen der Rezipienten abhängig, das Werk weise jedoch »universalistische Integrationstendenzen « (Böhler 1981: 370) auf, könne aber seine Wirksamkeit nur voll entfalten, wenn die Rezipienten den Konsens, auf den das Werk angelegt sei, für sich selbst vollziehen.

Dirk Skiba (Jena) berichtete von transkribierten Gesprächen zwischen chinesischen Studierenden der Germanistik und dem Autor Christoph Hein über Der Fremde Freund, Horns Ende und Die wahre Geschichte der Ah $Q$ in Shanghai sowie zwischen Studierenden der Universität Jena und dem Autor Tim Staffel über Terrordrom und Heimweh. Die Auseinandersetzung mit den Werken über das Gespräch mit Autoren eröffne anders als bei einer interkulturellen Hermeneutik, die sich neue Perspektiven auf die Texte verspreche, ausgehend von den subjektiven Lektüreerlebnissen Möglichkeiten des kulturellen Lernens, die es auszuloten gelte.

Abschließend berichtete Annegret Middeke (Plovdiv) über ein interessantes Projekt zwischen Plovdiver und Dresdener Germanisten, dessen Ziel es ist, ein Lehrwerk in Form eines Lese- und Arbeitsbuchs in deutscher Sprache zu erstellen. Das Lehrwerk soll sich auf die jeweiligen Fremd- und Eigenbilder in der deutschsprachigen und bulgarischen Literatur beziehen und damit auch die Disproportionalität der jeweiligen Wahrnehmung zum Thema machen. Für die bulgarische Literatur ging sie u.a. ausführlich auf Konstantinovs Roman Baj Ganju. Die unglaublichen Erzählungen über einen zeitgenössischen Bulgaren ein.
Themenschwerpunkt 2: Neue Methoden der Grammatikvermittlung

(Evelyn Müller-Küppers, Mainz; Inge Christine Schwerdtfeger, Bochum)

Die Vermittlung und das Lernen von Grammatik ist - Schlagworte wie »focus on form " und "postkommunikativer Fremdsprachenunterricht « deuten es an - heutzutage wieder sehr stark in das Interesse der Lehrenden und Lernenden gerückt. Dies wurde den Koordinatorinnen des Themenschwerpunktes $2 » N e u e$ Methoden der Grammatikvermittlung" sowohl im Vorfeld der Jahrestagung bewußt, gingen doch nach der Ankündigung der Tagung und ihrer Schwerpunkte wesentlich mehr Vortragsangebote ein, als in den dafür vorgesehenen elf »slots « untergebracht werden konnten, als auch auf der Tagung selbst, wo die für den Themenschwerpunkt vorgesehenen Räumlichkeiten zeitweise den Zustrom an interessierten Zuhörenden kaum fassen konnten.

Die Arbeit in diesem Themenschwerpunkt verfolgte das Ziel, »Methoden der Grammatikvermittlung und des Grammatiklernens vor dem Hintergrund neuerer kognitiver, emotionaler, linguistischer und didaktischer Konzepte zu beleuchten und, wenn möglich, Schwerpunkte dieser neuen Entwicklungen zu dokumentieren " (aus dem Ankündigungstext). Dem entsprechend hatten die Koordinatorinnen bei der Zusammenstellung des Programms darauf geachtet, daß möglichst unterschiedliche Ansätze zur Sprache kamen und die Mischung aus sowohl eher theoretischen als auch praxisorientierten Konzepten stimmte.

Gabriele Graefen (Institut für Deutsch als Fremdsprache/Transnationale Germanistik der Ludwig-Maximilians-Universität München) eröffnete den Themenschwerpunkt am Donnerstagnachmittag dann auch direkt mit einer kritischen Bestands- 
aufnahme und einem Plädoyer in Sachen »Veränderungen im mutter- und fremdsprachlichen Unterricht «. Die Referentin ging dabei auf die grammatikkritische Entwicklung in beiden Sprachdidaktiken ein, resümierte noch einmal den Verlauf der Debatte um Sinn oder Unsinn der Vermittlung von Sprachwissen an muttersprachliche oder fremdsprachliche Lernende seit den 70er Jahren und beleuchtete die aktuelle Situation unter Einbeziehung neuerer Ergebnisse der Sprachlehr- und -lernforschung. Mit Blick auf die zentrale Rolle des Verhältnisses von Linguistik und Didaktik plädierte sie abschließend für einen pragmatischen Umgang mit grammatischen Lehraufgaben und einen Unterricht, der durch einen funktionalgrammatischen Ansatz zu handlungspraktischem Sprachbewußtsein im Sinne von Angelika Redder verhilft.

Alle im Bereich Deutsch als Fremdsprache Lehrenden kennen und fürchten die berühmten Ad-hoc-Fragen im Unterricht, etwa der Art »Was ist denn genau der Unterschied zwischen ...«, oder »Warum kann ich denn hier ... nicht benutzen?«, auf die man entweder mit eben solchen Ad-hoc-Erklärungen reagiert, mit dem Risiko, sie später wieder revidieren zu müssen, oder die man mit dem ehrlichen Hinweis auf die nächste Sitzung verschiebt, da müsse man sich jetzt zu Hause selbst erst mal sachkundig machen. Während nun aber um die adäquate sprach- und grammatiktheoretische Fundierung einer Lernergrammatik $\mathrm{DaF}$ seit Jahren eine intensive Diskussion geführt wird, spielt die Frage nach dem grammatischen Wissen der Lehrenden bislang kaum eine Rolle. Offensichtlich wird nicht nur von den Lernenden vorausgesetzt, daß die Lehrenden als allwissende Instanzen über ausreichend systematisches, theoretisches und aktuelles grammatisches Wissen verfügen, was sich in der Praxis nun leider nicht bestätigt. So fragte Eva Breindl (Institut für Deutsche Sprache, Mannheim) in ihrem Beitrag zu Recht »Alle reden von der Lernergrammatik - und was ist mit den Lehrern?" und bot letzteren als Hilfe das über WWW zugängliche hypermediale Informationssystem GRAMMIS an, das auf Grund seiner medialen Ausgestaltung mit vielfältigen bequemen Zugangswegen zu grammatischem Wissen dem oben skizzierten Adressatenkreis sicherlich eine schnelle, systematisch-fundierte Information ermöglicht. Die theoretische Fundierung auf dem semantisch-funktionalen Konzept der Grammatik der deutschen Sprache (Zifonun; Hofmann; Strecker 1997) und des Handbuchs der deutschen Konnektoren kommt zudem dem in der DaF-Didaktik geltenden kommunikativen Paradigma entgegen. Breindl demonstrierte diese neue Art des Zugangs zu Grammatik am Beispiel der Konnektoren und sah sich in der Diskussion einer sehr interessierten Zuhörerschar gegenüber, der sie kompetent Rede und Antwort stand.

Mit dem nächsten Beitrag gerieten wieder mehr die Lernenden ins Blickfeld. Johannes Eckerth (Universität Hamburg) resümierte nämlich unter dem Titel "Grammatik- und Partnerarbeit - empirisch betrachtet « eine umfangreiche empirische Studie, in deren Zentrum die Frage stand, was in den Köpfen von Lernenden passiert, wenn sie im DaF-Unterricht gemeinsam Grammatikaufgaben bearbeiten. Bei diesen Aufgaben handelte es sich um eigens entwickelte, kommunikativ und sprachstrukturell orientierte TextReparatur-Aufgaben, untersucht wurden die bei der selbständigen gemeinsamen Bedeutungs- und Formaushandlung durch Lernerpaare in den Lernenden ablaufenden Lernprozesse und die erzielten Lernerfolge. Eckerth konnte die didaktische Gretchenfrage, ob denn Partnerbzw. Gruppenarbeit nun wirklich zu ge- 
genseitiger Hilfe oder aber zu Bestätigung und Perpetuierung von Fehlern führe, dahingehend positiv beantworten, daß die von ihm dokumentierten Ergebnisse auf vielfältige Lernprozesse, eine stärkere Bewußtheit der Lernenden für den eigenen Lernprozeß, eine Sensibilisierung für zielsprachliche Wissensbestände schließen lassen. Die Dissertation des Referenten (Fremdsprachenerwerb in aufgabenbasierten Interaktionen, Eckerth 2002) wird die bislang etwas dünn besiedelte Forschungslandschaft im Bereich »empirische Erkenntnisse zu Partner-/ Gruppenarbeit« zweifellos bereichern.

Michael Koenig (Universität Kassel) nahm in seinem Hauptvortrag: »Attention please! - oder worauf man im Grammatikunterricht auch noch achten sollte" direkten Bezug auf das gegenwärtig nachhaltig steigende Interesse am Grammatikunterricht, das in den Beiträgen des Vortages bereits augenfällig geworden war, indem er zunächst einen breit angelegten Bericht über den gegenwärtigen Forschungsstand des "focussing " und »noticing " als Strategien des Grammatiklehrens und -lernens gab. Danach lenkte er den Blick auf den Lernenden, dessen individuelle Faktoren als bestimmend für den grammatischen Lernprozeß isoliert wurden. Diesen unterschiedlichen individuellen Faktoren entsprechend müssen verschiedene Wege gefunden werden, das »noticing « von grammatischen Besonderheiten der Lernenden zu schärfen. Grammatikerwerb kann, so Koenig, nur erreicht werden durch ein subtiles $\mathrm{Zu}$ sammenspiel von "noticing « der Lernenden und »focussing « in den Lehrmaterialien. Wie dieses vollzogen werden kann, wurde an zahlreichen Beispielen aufgezeigt, die sich nicht nur auf das Deutsche als Fremdsprache bezogen.

Eine bisher fehlende Dimension brachten die Referentinnen Eva-Maria Willkop
(Universität Mainz) und Maria Thurmair (Universität Regensburg), mit ihrem Beitrag: »Goldene Eier?! - Die Textgrammatik der deutschen Sprache" in die Sektion ein: die Vorstellung einer Grammatik. In die Textgrammatik der deutschen Sprache wurde von deren Mitverfasserinnen zunächst durch eine Darlegung von deren methodischen Leitprinzipien eingeführt, die durch vielfältige Beispiele illustriert wurden. Hierbei wurden insbesondere die Einbeziehung der Leiblichkeit in die semantischen Beschreibungen und das dialogische Prinzip als zentrale Bausteine der Textgrammatik herausgearbeitet. Die Referentinnen leiteten ihre Ausführungen über in höchst anschauliche Didaktiktisierungsvorschläge zweier grammatischer Phänomene, zum einen Pronomina, zum anderen das Tempussystem. Der nächste Beitrag von Anne Vorderwülbecke (Universität Heidelberg): »Lebendige Grammatik - mehr Körpersprache - weniger Metasprache « fügte sich dem vorangehenden durchaus überzeugend an. Ihr Ziel, Lernenden mit unterschiedlichen Lernhaltungen Wortstellungsregularitäten der deutschen Sprache durch Gesten, Realia, Bilder und Leitwörter zu vermitteln, wurde den Zuhörern in einer lebendigen Präsentation veranschaulicht.

Einen in der Vortragsreihe bisher nicht angesprochenen Aspekt des heutigen Standes der Grammatikforschung erschloß Thomas Wagner (Dublin City University) mit seinem Beitrag: »Wie lernt man Grammatik ohne Regeln? Der Umgang mit Grammatik in Deutsch als Fremdsprache vor dem Hintergrund neuerer kognitiver linguistischer Forschung «. Die Pluralbildung des Deutschen und die starken Verben als Beispiel nutzend, stellte Wagner Modelle kognitiver linguistischer Forschung vor (z.B. den Dualen Mechanismus und den Konnektionismus). Hiermit sollte der Frage nachgegangen werden, ob aus diesen Modellen mögliche Erklärungsan- 
sätze dafür abgeleitet werden können, auf welche Weise Lernende von Sprachen Zusammenhänge erschließen, für die nur ein unzureichender Regelapparat vorliegt.

Diese kognitiven Modelle, so zeigte Wagner auf, sind deshalb so interessant für an grammatischer Konstruktion Interessierte, weil sie »für den Umgang mit Irregularitäten in gewisser Weise Sprachtransfer voraussagen «. Die Resultate wurden an Kategorisierungen, die Lernende englischer irregulärer Verben vollziehen, gespiegelt. Überlegungen zur möglichen Erklärungsstärke von Modellierungen in der kognitiven Linguistik für die Klärung des Konzepts »Regelwissen im Grammatikunterricht « beschlossen diesen Vortrag.

Die anschließende Präsentation von $\mathrm{Sa}$ bine Dinsel (München) hatte phasenweise den Charakter eines workshops. Sie zeigte in ihrem Beitrag: »Grammatiklernen leicht(er) gemacht « vielfältige Grammatikübungen für Lernende vor allem im Anfangsunterricht, wobei sie die Teilnehmenden motivierte, bei den Übungen mitzutun.

Dieser Tag wurde beschlossen durch den Vortrag von Mathilde Hennig (Szeged/ Timisoara). In ihrem Beitrag: »Wie kommt die Grammatik der gesprochenen Sprache in den Unterricht Deutsch als Fremdsprache?" lenkte sie die Aufmerksamkeit auf ihre Forschungen zu den Grundbedingungen prototypischer gesprochener Sprache, um im Anschluß daran Überlegungen vorzustellen, wie eine Grammatik des Systems und eine Grammatik der Norm der gesprochenen Sprache in ihrer gegenseitigen Verschränktheit im Unterricht Deutsch als Fremdsprache ihren Platz finden können.

Im bunten Reigen vielfältiger Blickrichtungen auf die derzeitigen Methoden der Grammatikvermittlung sollte die Außenperspektive nicht fehlen. Und so ging am
Samstagvormittag der Blick ins Nachbarland Dänemark, wo man nach den Ausführungen von Peter Colliander (Wirtschaftsuniversität Kopenhagen) »Neue Wege der Grammatikvermittlung " beschreitet. Der Referent beklagte zunächst den deutlichen Niedergang grammatischer Kenntnisse bei Lehrenden und Lernenden von Deutsch als Fremdsprache an Schulen und Gymnasien in Dänemark, was er auf die fehlende Motivation der Dänen für die Beschäftigung mit dem sprachlichen System, den Autoritätsschwund und Generationenkonflikt zwischen Lehrern und Schülern zurückführte. Colliander stellte am Beispiel der Umstrukturierung des Grammatikunterrichts im Deutschstudium an der Wirtschaftsuniversität Kopenhagen (Bachelor-Studiengang) Ansätze vor, dieser Situation abzuhelfen. Dabei fiel auf, daß er großen Wert auf die Vermittlung des grammatischen Systems, also Wissen über die Sprache, legte und die Inhalte der von ihm angebotenen Lehrveranstaltungen eher den Charakter einer Einführung in die Linguistik hatten. Das Beherrschen sprachlicher Formen und Regularitäten wurde in häusliche Drillübungen verlegt.

Der Beitrag von Barbara Schmenk (RuhrUniversität Bochum) beschloß am Samstag den Themenschwerpunkt. Mit dem Titel »Passiv ist nicht nur Passiv. Grammatik in einem narrativen dramapädagogischen DaF-Unterricht « hatte die Referentin noch einmal eine große Zuhörerschaft angelockt, sei es nun durch das LieblingsGrammatikthema vieler DaF-Lehrender oder durch die Perspektive, eben dieses Standardthema einmal didaktisch-methodisch ganz anders vermittelt zu sehen. Entsprechend groß war auch die Zustimmung, als Barbara Schmenk eingangs auf die Beobachtung einging, daß sowohl Lernende als auch Lehrende das Passiv im Deutschen häufig für schwer halten 
und der erwünschte produktive und rezeptive Transfer trotz eines umfangreichen Übungsangebots in Lehrwerken, ergänzt durch seitenlange Zusatzübungen der Lehrenden, ausbleibt. Hier nun setzte die Referentin mit dem Konzept an, nicht die formalgrammatischen Regelmäßigkeiten des Passiv zum Schwerpunkt zu machen, sondern im Sinne der anthropologisch-narrativen Didaktik von I. C. Schwerdtfeger das Leiblichkeitskonzept (Lernen im Sinne von "Einverleiben«) von subjektiv bedeutungsvollen Erfahrungen (»Ich bin nicht aktiv, mit mir geschieht etwas") ausgehend, über die metaphorische Konzeption von Passivität bis hin zur Realisierung in entsprechenden sprachlichen Konstruktionen das Passiv als »einverleibte Bedeutung ", als "persönlichen Bedeutungskern" erfahrbar zu machen. Dabei griff sie auch auf Aspekte der Dramapädagogik im Unterricht Deutsch als Fremdsprache zurück.

Noch im Verlauf der Tagung konnten die Koordinatorinnen aus entsprechenden Rückmeldungen schließen, daß der Themenschwerpunkt eine insgesamt zufriedene Zuhörerschaft gefunden hatte.

\section{Themenschwerpunkt 3: Einheit von Fach- und DaF-Unterricht}

(Peter Dammeier, Köln; Marie-Luise FreinPlischke, Kaiserslautern)

Klaus Baumann (Universität Leipzig) stellte in seinem Beitrag »Die interdisziplinäre Entwicklung fachkommunikativer Kompetenz im DaF-Unterricht" dar, welche Teilkompetenzen in der Vermittlung berufsbezogener Kommunikationskompetenz zu unterscheiden sind. Der Beitrag zeigte die breite Spanne berufsbezogener Kommunikationskompetenz, die von der interkulturellen und sozialen Kompetenz über die Teilkompetenz des Fachdenkens, die fachliche, funktionale, textuelle, stilistische, textsyntaktische bis hin zur lexikalisch-semantischen Teilkompetenz reicht.

Es wurde deutlich, daß fachbezogener Fremdsprachenunterricht auf der Basis eines ganzheitlich funktionierenden Systems dieser Teilkompetenzen erfolgen muß. Kulturspezifische Unterschiede des Fachkommunikationsstils - z. B. hinsichtlich der Exkurse in Fachtexten, des Bezuges auf Aussagen wissenschaftlich anerkannter Autoritäten oder Gliederungstechnik - sind ebenso zu berücksichtigen wie die Teilkompetenz des Fachdenkens, die über Denkstrukturen und gedankliche Modelle die Kommunikationskompetenz beeinflußt.

Am Beispiel der Textsorte fachbezogener Essays wurde erfaßbar, wie sehr die textuelle Teilkompetenz als Wissen über die Makrostruktur eines Textes das Erfassen der im Text enthaltenen Informationen befördert. Die textsyntaktische Teilkompetenz, die umschreibt, in welchem Umfang syntaktische Elemente in verschiedenen Fachtexten auftreten, ist ebenso zu vermitteln wie die Bedeutung der Termini der Fachkommunikation im fachbezogenen DaF-Unterricht als Gegenstand der lexikalisch-semantischen Teilkompetenz. Es wurde deutlich, welch vielfältige Kompetenzen notwendig sind, um Lerner zur Kommunikation im Fach zu befähigen. Dies gilt es bei Didaktisierungsbemühungen zu berücksichtigen.

Rupprecht S. Baur (Universität Essen) behandelte mit seinem Beitrag »Zum Erwerb und zur Vermittlung von Fachsprachen im Unterricht Deutsch als Fremdsprache" anhand von Video-Ausschnitten am Beispiel einer ausgesiedelten Schülerin (7. Schuljahr) sprachliche Phänomene bei der Vermittlung eines Fachtextes Biologie (Pawlow-Effekt). Ausschnitte aus dem Förderunterricht machten deutlich, wie sehr allgemeine und fachspezifische Textarbeit ineinandergreifen. Nicht nur fachspezifi- 
sche Lexik, sondern auch nicht zum Fachwortschatz gehörende Lexik wurden semantisiert. Deutlich wurde auch, daß sich der Unterricht nicht auf die Lexik beschränken darf, die der Lerner erfragt; der Lehrer muß vielmehr wichtige Begriffe absichern, auch wenn diese nicht erfragt werden, da Schüler u.U. Verstehensschwierigkeiten nicht immer ausreichend thematisieren können. Eine weitere Schwierigkeit dieses Unterrichts stellt die Überprüfung von Verstehensleistungen dar, da diese über mündliche oder schriftliche Produktionen abgeprüft werden, d.h. weitere Fertigkeiten erfordern. Das Ineinandergreifen von rezeptiven und produktiven Fertigkeiten macht eine Beurteilung von Verstehensleistungen schwierig.

Eva Lipkowski (Universität Essen) stellte mit ihrem Beitrag "Zur Vermittlung der sprachlichen Seite fachlichen Lernens" »Förderunterricht für Kinder und Jugendliche mit Migrationshintergrund « vor, der von Studierenden der Universität Essen - betreut von universitären Mitarbeitern - erteilt wird. Dargestellt wurden hier die sprachlichen Probleme der Schülerinnen und Schüler in den Fächern Deutsch, Mathematik und Englisch, die Erfahrungen der Förderlehrkräfte hinsichtlich der sprachlichen Probleme ihrer Schüler und Schülerinnen (z.B. Orthographie, grammatische Strukturen) und schließlich der Umgang der Schule mit diesen Problemen. $\mathrm{Zu}$ den Problemfeldern, die die Schüler und Schülerinnen benennen, gehören insbesondere die Lektüre und das Verstehen literarischer Texte, wobei in diesem Zusammenhang auch die Auswahl der Texte durch die Lehrer thematisiert werden müßte. Im Fach Mathematik sind Verstehensschwierigkeiten insbesondere bei Textaufgaben erkennbar. Ein weiteres Problemfeld stellen schriftliche Tests dar, die die Problematik aufzeigen, daß Verstandenes nicht adäquat zu Papier ge- bracht werden kann. Es wurde deutlich: Eine Kooperation zwischen Schule und Universität, eine sprachdidaktische Ausund Fortbildung für Studierende, aber auch Fachlehrer scheint angezeigt.

Ingeborg Hofmann (Universität Greifswald) stellte in ihrem Referat »Einführung in das deutsche Recht - Koordination juristischer und sprachlicher Aspekte in einem Sokrates-Programm " ein Programm der Rechts- und Sprachwissenschaftlichen Fakultät der Universität Greifswald vor. Dort werden seit mehreren Jahren vom Lektorat Deutsch als Fremdsprache Studierende aus Sokrates-Programmen 14tägige Einführungskurse in das deutsche Recht sowie weiterführender Unterricht im Semester (2 Stunden wöchentlich) angeboten. Die Textbasis - erarbeitet in Kooperation mit Kollegen aus der juristischen Fakultät - bilden juristische Originaltexte, schriftliche wie mündliche. Das Textkorpus umfaßt argumentative, deskriptive, narrative und referierende Texte aus Vorlesungsskripten, Fachzeitschriften, Lehrbüchern und Gesetzestexten. Das Textkorpus konstituiert sich also auf der Basis von studienrelevanten fachlichen Inhalten des Jura-Studiums. Anhand dieser Texte werden u.a. in der juristischen Fachsprache frequente Textsorten, sprachrelevante Handlungen (z. B. Definieren) und Sprachmittel (morphologische, syntaktische Elemente) bewußt gemacht. Neben der Erarbeitung von Termini werden schließlich auch Unterschiede in Rechtssystemen (inhaltliche und sprachliche Phänomene) erarbeitet, wobei nicht selten sprachliche Entsprechungen fehlen, was deshalb eine besondere Schwierigkeit darstellt, da rechtsvergleichende Fachwörterbücher nur unzureichend verfügbar sind. Das Ziel dieser Kurse ist es, den Studierenden durch die Didaktisierung von fachtypischen juristischen sprachlichen Mitteln, Handlungen, Kommunikationsweisen den $\mathrm{Zu}$ - 
gang zu und Umgang mit juristischen Fachtexten zu erleichtern und sie so zum Diskurs im Fach zu befähigen.

Niamh O'Mahony und Daniela Hartmann (Aston University, Birmingham) verdeutlichten mit ihrem Beitrag »Manager auf Probe: Organisationstheoretische Ansätze als Grundlage für die Unterrichtspraxis Wirtschaftsdeutsch « anhand konkreter Unterrichtsbeispiele, wie auf der Grundlage organisationstheoretischer und soziopsychologischer Ansätze DaF-Lerner (Zielgruppe: 18-22 Jahre, Studium BWL/ Deutsch) in Beurteilungsvorgänge in den Unterrichtsprozeß eingebunden werden können. Nach einleitenden Grundgedanken zu Fragen des Gruppendrucks und des Manageralltags stellten sie ein 3-Phasen-Modell zur Integration von BottomUp-Prozessen im Wirtschaftsdeutsch-Unterricht vor. Vorgestellt wurde das Projekt »Marketing «: Zum Ende des 2. Semesters wird ein Marketing Szenario präsentiert. In einem Zeitrahmen von 3 Monaten ist von Studierenden in Teamarbeit nicht nur ein Marketing-Plan für die Positionierung eines Produkts auf dem britischen Markt $\mathrm{zu}$ erarbeiten und in Referaten vorzustellen; es ist darüber hinaus auch ein Kriterienkatalog zur Evaluation von Präsentationen zu erstellen. Das Besondere und die Vorzüge dieses auf Szenarien basierenden Unterrichts wurden deutlich: Die klassischen Lehrer/Lerner-Rollen werden aufgebrochen, der Unterricht hebt klar auf die Bedürfnisse der Zielgruppe ab.

Klaus Knödlmayr (Studienkolleg München) stellte mit dem »Projekt Deutsch Fachsprache in Chemie/Biologie (MedizinerKurs an Studienkollegs) « einen fachsprachlichen Zusatzunterricht vor, der am Studienkolleg München für den medizinisch/biologischen Kurs (M-Kurs) ergänzend zum Chemie/Biologieunterricht erteilt wird. In diesem Kurs werden 30 Stunden Unterricht durchgeführt, davon 1/3 Chemie/Biologie- Unterricht,
$1 / 3$ Deutsch-Unterricht und $1 / 3$ sonstiger Unterricht. Für den fachsprachlichen Zusatzunterricht ist eine Doppelstunde pro Woche reserviert, unterrichtet vom jeweiligen Chemie/Biologie-Fachdozenten. Ziel dieses Zusatz-Unterrichts ist es, das Augenmerk der Studierenden auf den Wert der sprachlichen Qualität ihrer Prüfungsarbeiten auch in den Sachfächern $\mathrm{zu}$ lenken und sie zu befähigen, auch komplizierte fachliche Sachverhalte angemessen zum Ausdruck zu bringen. Die Unterrichtsthemen werden so gewählt, daß sie die Themen des Fachunterrichts vertiefen und ergänzen. Der Schwerpunkt des Unterrichts liegt auf der Textproduktion. Neben dem Bearbeiten von Texten aus Facharbeiten werden insbesondere Texte zu schwierigen Themen aus dem Fachunterricht erstellt. An Beispielen, z. B. der Destillation von Rotwein, wurde der Unterrichtsverlauf aufgezeigt: Nachdem auf der Grundlage des Fachunterrichts fachliche Zusammenhänge geklärt sind, erarbeiten die Studierenden in Gruppen einen Text zu einer gegebenen Aufgabe, der anschließend vorgetragen und besprochen wird. Auf dieser Grundlage wird u. a. ein im Fachunterricht häufig benutzter Grundwortschatz (Substantive, Verben, Adjektive, Wendungen) erarbeitet. Ein gemeinsam produzierter Mustertext bildet den Abschluß. Eine Erfolgskontrolle findet dadurch statt, daß in Chemie/ Biologie-Prüfungen eine Aufgabe diesem Zusatz-Unterricht entstammt. Diese Aufgabe geht dann entsprechend dem Stundenanteil des Zusatzunterrichts am Gesamtunterricht $(1 / 7 ; 1 / 6)$ in die Benotung ein, wobei sprachliche und inhaltliche Elemente im Verhältnis 1:1 berechnet werden. Da dieser Zusatz-Unterricht bei den Studierenden auf große Resonanz stößt, wurden mittlerweile ähnliche Projekte auch im W- und G-Kurs gestartet. 
Themenschwerpunkt 4: "Integration von Online- und Präsenzlernen «

(Matthias Jung, Düsseldorf)

Im Mittelpunkt des Themenschwerpunktes 4 sollte nicht das Thema »Internet« bzw. »Neue Medien« allgemein stehen, sondern speziell Konzepte und Beispiele zur Verbindung von Präsenz- und Online-Lernen bzw. von starren Lernprogrammen und individueller Unterstützung durch Online-Tutoren. Denn die Erkenntnis, daß dies die effizientere Form des Tele-Lernens ist, setzt sich mittlerweile auch bei Nicht-Pädagogen durch (Stichwort »E-Learning « bzw. genauer »blended learning « speziell für integrierte Konzepte).

Natürlich steht und fällt das Online-Lernen auch mit der (Übertragungs-)Technik. Obwohl es hier in den letzten Jahren große Fortschritte gegeben hat, was Geschwindigkeit und Möglichkeiten des Datentransfers über das Internet angeht, machen leider die Zuverlässigkeits- und Kompatibilitätsprobleme der besten Pädagogik immer noch zu häufig einen Strich durch die Rechnung. Das spiegelte sich auch in den Vorträgen des Themenschwerpunktes wider, in dem einige Vorträge durch technische Probleme bei der Präsentation erheblich beeinträchtigt wurden.

Einleitend gaben Matthias Jung (Düsseldorf) und Jürg Roche (München) einen Überblick über die fremdsprachendidaktischen Entwicklungen, die sich hinter der Propagierung des integrierten OnlineLernens verbergen. Josef Bornhorst (München) informierte zunächst über jetzt an den Start gehende Online-Lernprojekte des Goethe Instituts Inter Nationes. Im weiteren konzentrierte er dann seine Darstellung auf den Online-Sprachkurs $» R e-$ daktion D ", der allerdings im wesentlichen ein auch über das Internet zu absolvierender Selbstlernkurs auf CD bleibt.
Anschließend stellten Jörg Roche, Stephan Schlickau und Iris Zendath (alle München) Evaluationskriterien für Lernsoftware und Internetübungen zur Diskussion. Helmut Frielinghaus (Hamburg) zeigte, wie man mit Hilfe einer sogenannten Lernplattform feste Module und individuelle Lernformen im Internet organisieren kann. Susanne Vitz-Manetti (Padua) berichtete von immerhin 3 Jahren Erfahrung mit der Verbindung von OnlineSoftware und universitärem Fremdsprachenunterricht, wobei sie ein nicht unkritisches, aber grundsätzlich positives Fazit zog.

Am Freitag berichtete Nicola Würffel (Gießen) von ihren empirischen Untersuchungen und konnte an Fallbeispielen deutlich machten, warum Lerner oft bei der Aufgabenbewältigung am Bildschirm scheitern. Dierk Hoffmann (Colgate, USA) hielt ein engagiertes Plädoyer für Online-Videokonferenzen im DaFUnterricht, das mit schönen Beispielen aus seiner großen diesbezüglichen Praxis illustriert wurde. Stephan Schlickau (München) beschäftigte sich vor allem mit dem interkulturellen Potenzial von VideoKonferenzen über Grenzen hinweg.

Den Samstag eröffnete Claudia Popov (Leipzig), die in Eigenregie eine vielschichtige Website zum Deutschlernen betreibt und darüber seit mehreren Jahren erfolgreich Einzelunterricht per EMail und Konferenzsoftware abwickelt. Abschließend stellte Fritz Frey (Bremen) ein Konzept zum kreativen Schreiben mit Wechsel von Präsenzphasen und gemeinsamem Lernen im Netz über eine Lernplattform vor, das im Wintersemester umgesetzt werden soll. Abgeschlossen wurde der Themenschwerpunkt dann mit dem Plenarvortrag von Peter Liddell (Victoria, Kanada), der seine Einschätzung der aktuellen Entwicklung des Online-Lernens im DaF-Bereich gab. 
Die Beiträge des Themenschwerpunktes zeigten, daß integrierte Konzepte im Bereich des DaF-Lernens noch ziemlich am Anfang stehen. Die Verbindung von unpersönlichen Lernprogrammen und interpersonalem Lernen - sei es 1:1 mit einem Online-Tutor oder als »kollaboratives Lernen « mit einer Lerngruppe, sei es am Bildschirm oder in einer Gruppenpräsenzphase - ist trotz einiger programmatischer Diskussion zum »integrierten Lernen « noch kaum verwirklicht. Immerhin konnten von den wenigen Lehrerfahrungen, die hier derzeit für den DaF-Unterricht vorliegen, einige im Themenschwerpunkt vorgestellt werden. Und einige der Vortragenden machten deutlich, daß Online-Lehren und -Lernen auch Spaß machen kann.

Der grundsätzliche Trend zu mehr Online-Lernen ist aus vielen Gründen ungebrochen und kann nicht als Mode-Erscheinung abgetan werden - genau deswegen sind jetzt auch didaktische Konzepte und praktische Erfahrungen mehr als rein technische Expertise und Begeisterung gefragt.

\section{Forum Deutsch als Fremdsprache (Gisela Schroth, Düsseldorf)}

Krystyna Kudlinska-Stankulova, Sofia; Regine Hartung, Hamburg: Sprachen öffnen Welten (= Heft 7 der Reihe »Miteinander leben in Europa «)

Zum Auftakt des Forums DaF stellten die beiden Referentinnen ihr Heft 7 der Reihe »Miteinander leben in Europa « vor. Die erste Veranstaltung war noch relativ schwach besucht, was sich aber schnell änderte.

In »Miteinander leben in Europa « sollen Sprachlernerfahrungen, die eigene Einstellung zu Sprache und Sprachenpolitik und Politik durch und über Sprache thematisiert werden. Unter dem Titel Sprachen öffnen Welten bietet die bulgarisch- deutsche Arbeitsgruppe an, über die eigene Sprachlernbiographie zu reflektieren, den Sprachgebrauch, die Wichtigkeit von Sprache in Abhängigkeit von verschiedenen sozialen Sphären bewußt zu machen. Der Beitrag präsentiert Ergebnisse aus der unterrichtlichen Erprobung.

Gunde Kurtz, Kaiserslautern: "Grammatik im bedeutungsbasierten Sprachenlernen "

Die Referentin stellte dar, wie mit rein funktional orientiertem Umgang mit authentischem Sprachmaterial nicht nur die Toleranz der Lernenden gegenüber der Menge an unklar Bleibendem überfordert wird, sondern daß man damit auch Chancen für den sorgfältigen Aufbau produktiver Sprachkompetenz vergibt. Für dieses Problem stellte sie als Lösungsvorschlag vor, wie man im Sprachunterricht grundsätzlich von Bedeutungen ausgehend nicht nur inhaltliche, sondern auch formale Eigenheiten von Texten und Strukturen in der Zielsprache erlernen lassen kann. Wie dies aussehen kann, speziell wie strukturelle Eigenschaften von Sprache konzentrisch-bedeutungsorientiert vermittelt und gelernt werden, zeigte Frau Kurtz an einigen Beispielen auf. Bei diesem Ansatz werden in der Anfangsphase Ankerpunkte gesetzt, an denen nach und nach immer komplexere Erscheinungsformen der Zielsprache festgemacht werden können, so daß die Lernenden einen kontinuierlichen Aufbau einer überschaubaren Menge von in sich konsistenten Sprachbereichen erleben.

Verena Katz und Sigrun Schroth-Wiechert, Hannover: „Die DSH und projektorientierter Unterricht: Utopie oder Chance?"

Frau Katz und Frau Schroth-Wiechert stellten in ihrem Referat dar, welche Schritte sie in Richtung DSH-Differenzierung unternommen haben. Im Rahmen des Vorbereitungsunterrichts auf die DSH 
haben sie im Wintersemester 2001/2002 am Fachsprachenzentrum der Universität Hannover ein Projekt durchgeführt, das im wesentlichen darauf basiert, daß die ausländischen Studierenden in Gruppen entsprechend der von ihnen gewählten Fachrichtungen zusammengefaßt werden und in diesen Teams ein übergeordnetes Thema fächerorientiert bearbeiteten. Im Forum DaF stellen sie die Ergebnisse der einzelnen Projektarbeiten des zurückliegenden Semesters vor.

Nach einem Rückblick, bei dem die Vorbereitung, die Durchführung und die Ergebnisse dieses ersten Projektdurchlaufes skizziert wurden, gingen die Referentinnen auch auf die zentrale Frage nach dem Stellenwert eines projektorientierten Ansatzes im DSH-Bereich ein. Die Ergebnisse des Projekts haben eine Motivationssteigerung "auf beiden Seiten « sowie eine Verbesserung des schriftlichen Teils der DSH ergeben.

Klaus Vorderwïlbecke, Mannheim: »Das Lernsystem ProGr@mm»

Klaus Vorderwülbecke erläuterte die verschiedenen Komponenten der onlineGrundgrammatik ProGr@mm (kurz für: Propädeutische Grammatik) und stellte die bisher erarbeiteten Module kurz vor. ProGr@mm wird als Lernsystem entwikkelt, das speziell auf die universitäre Lehre zugeschnitten ist. Inhaltlich basiert es auf dem vom Institut für Deutsche Sprache entwickelten grammatischen Informationssystem GRAMMIS, enthält aber zusätzlich orientierende Einleitungstexte, Übungen und Aufgaben und auch neue Texte. In einem späteren Stadium wird es, wie der Referent berichtete, auch "geführte Touren « durch die Grundgrammatik des Deutschen geben. Dank dieser didaktischen Ausrichtung ist es nicht nur im muttersprachlichen Bereich, sondern auch in Kursen für fortgeschrittene DaF-Lernende einsetzbar.
Luca Lauga-Engelbert, Düsseldorf: »Vom engagierten Gruppenlehrer zum professionellen Einzeltrainer«

Gängige Ausbildungen für Sprachlehrkräfte gehen alle von der Situation des schulischen Gruppenunterrichtes aus. Wie Luca Lauga-Engelbert darstellte, sind die dabei vermittelten Fertigkeiten aber nur begrenzt auf Sprachtrainings im Auftrag von Firmen oder Institutionen übertragbar. Immer mehr Sprachschulen und Institute gehen dazu über, ihre Sprachtrainings in die Firmen zu verlagern, wobei diese meist in Form von Einzelbetreuung oder Kleingruppen stattfinden. Die Referentin zeigte auf, daß und wie sich Anforderungen an einen professionellen Sprachtrainer verändert haben. Der Sprachtrainer hat nun einen geschäftlichen Auftrag, er bietet einen Service an. Der "Schüler« ist der Kunde, und der »Unterricht « wird so zu einem geschäftlichen Treffen, wobei beide Seiten Einfluß auf den Ablauf nehmen. Der Lehrer wird zum Trainer, der neue Trainingsformen in einer Firma entwickeln muß, die die spezifische Situation der Teilnehmer optimal berücksichtigt.

Elisabeth Neurohr und Ingrid Plank, München: »Verben mit Präpositionen «

Verben mit Präpositionen sind ein Thema, das in der Linguistik, vor allem aber in Lehrwerken und wohl auch in der Unterrichtspraxis, meist vernachlässigt wird. Es sollte aber, so meinen die Verfasserinnen, im Unterricht für fortgeschrittene Anfänger gleichberechtigt neben anderen grammatischen Schwerpunkten stehen. Anhand von zum Thema konzipierten und im Unterricht erprobten Arbeitsblättern veranschaulichten sie die sprachpraktische Arbeit und zeigten auf, welche Argumente dafür sprechen und welche didaktischen Anforderungen sich für eine systematische und progressive 
Einführung und Übung von Verben mit Präpositionen ergeben.

Jin Zhao, Marburg: »Warum ist das Hörverstehen schwer für chinesische Deutschlerner?«

Da Zhin Zhao kurzfristig erkrankt war, hielt an ihrer Stelle Wolfgang van Straelen das Referat bzw. stellte die Ergebnisse einer von Frau Zhao durchgeführten Studie dar mit dem Ziel herauszufinden, warum so viele Chinesen erhebliche Probleme beim Hörverstehen haben. Frau Zhao hatte diese empirische Untersuchung angesichts der Tatsache, daß eine wachsende Anzahl von Chinesen an Sprachkursen in Deutschland teilnimmt, unter den Deutschlernern im IZS (Interkulturelles Zentrum für Sprache und Kultur) an der Philipps-Universität Marburg durchgeführt, um den Lehrenden sowie den Lernenden zum besseren Lehren bzw. Lernen zu verhelfen. Dabei ergaben sich vor allem der Einfluß der Muttersprache, die Lerngewohnheiten aus der schulischen Sozialisation, die fehlenden Kenntnisse über Hörstrategien sowie die mangelhafte Beherrschung von Arbeitstechniken als Gründe.

Andreas Tomaszewski, München: »Vorstellung der em-Übungsgrammatik für die Mittelstufe (Max Hueber Verlag)«

Andreas Tomaszewski stellte die emÜbungsgrammatik (2002) von Hering, Matussek, Perlmann-Balme vor. Sie ist nach folgendem Konzept aufgebaut: Der
Stoff der deutschen Grammatik (Mittelstufe) ist in lernergerechte, überschaubare und in einem bestimmten Zeitraum zu bewältigende Einheiten aufgeteilt immer von den Hauptschwierigkeiten und -fehlerquellen der Lernenden aus betrachtet. Diese Einheiten werden auf einer Doppelseite präsentiert: auf der linken »Darstellungsseite « die Strukturen und Regeln, auf der rechten »Übungsseite« vielfältige Übungen zu dem jeweiligen Phänomen, die sich an authentischen Texten orientieren.

Susanne Even, Arlington (USA): »Kognitives und affektives Lernen im dramagrammatischen Unterricht: Lernstile, Lernstrategien und Lernanschauungen "

Susanne Even klärte zunächst die Begrifflichkeit. Dramagrammatik, auch dramapädagogischer Grammatikunterricht, ist darauf angelegt, Grammatik im Handlungsvollzug $\mathrm{zu}$ realisieren und gleichzeitig eine positive Lernhaltung zur Grammatik zu fördern. Mit Methoden aus den dramatischen Kunstformen wird sich der fremdsprachlichen Grammatik auf kreative, ästhetische, emotionale und kognitive Weise genähert. Damit werden intensive und nachhaltig wirksame Erfahrungen mit der fremden Sprache und ihrer Grammatik ermöglicht. Even zog eine Vielzahl von Feedbacks aus Teilnahme- und Beobachterperspektive heran, wobei sie sich sowohl auf Langzeitstudien als auch auf Einzelworkshops bezog. 